\title{
A Method of Map Matching based on Particle Filter in Indoor Positioning
}

\author{
Zhongliang Deng, Fengli Ruan ${ }^{a}$, Shunbao Lu, Ruoyu Zheng, Hui Zeng, Yeqing \\ Fang and Yi yang
}

School of Electronic Engineering, Beijing University of Posts and Telecommunications, Beijing 100876, China

aruanfenglijiayou@163.com

Keywords: Indoor Positioning, Map matching, Sensor, Particle filter.

\begin{abstract}
Map information and sensor information is considered to be an effective way to assist indoor wireless location. Wireless location combined with map information and Sensor information can solve many problem such as positioning point cross the wall, falling into the wrong room. In this paper, a new indoor map matching algorithm was proposed based on particle filter. The indoor positioning system could be considered as non-linear system, so Non-parametric Monte Carlo (Monte Carlo) simulation method could achieve recursive Bayesian filtering, at the same time, the indoor GIS data, and sensor information, wireless location information was incorporated into the weight value of the particles to revise the observations constantly. Many experiments show that the indoor positioning accuracy is improved.
\end{abstract}

\section{Introduction}

Currently, the accurate indoor positioning was relayed on the Wi-Fi positioning technology, However, its positioning error is generally 3-8 meters and the positioning accuracy is very difficult to break through[1].under the commercial environment, m-level positioning accuracy cannot specific room and the corridor, and as to the indoor map more finely than the outdoor map, the jitter on the positioning result was reflected more obvious indoor map, leading to poor user experience. In the case that indoor wireless positioning accuracy was limited, the map matching combined with sensor information and map information is an effective way to improve indoor positioning accuracy. Map matching is widely used in outdoor car navigation system, using the road network information to improve the vehicle positioning accuracy. The interior building structure model was build based the indoor base vector information, and the simple geometric matching algorithm was used to constraint the wireless location results, however, this algorithm does not consider the process a smooth positioning results filtering problem. Due to the non-parametric characteristics, the particle filter get rid of the problem solving nonlinear filtering Gaussian random variable must satisfy the constraints [2]. It is an effective solution to analysis nonlinear dynamic systems [3].

In order to reduce the indoor wireless positioning error, this paper proposed a new indoor map matching algorithm based on particle filter. Firstly, the principle of particle filter was introduced. Secondly, indoor map topology model was build and PDR (Pedestrian Dead reckoning) information was used from the mobile phone sensor. Finally, the algorithm was validated by experiments, and the result showed that the algorithm improved the indoor positioning accuracy.

\section{The particle filtering principle}

The core of particle filter is using the large number of randomized trials to approximate the posterior probability distribution, and using the sample mean to replace the integration operation to obtain the minimum variance distribution state. A dynamic system model can be described by the following equation:

$$
\begin{aligned}
& x_{k}=f\left(x_{k-1}, v_{k-1}\right) \\
& z_{k}=h\left(x_{k}, w_{k}\right)
\end{aligned}
$$


The equation (1) is the state equation of the system, and the equation (2) is the observation equation of the system. $x_{k}$ is the system state vector at $\mathrm{k}, z_{k}$ is the observation value, $v_{k-1}$ and $w_{k}$ are the noise. The initial probability density is the equation (3):

$$
p\left(x_{0} \mid z_{0}\right)=p\left(x_{0}\right)
$$

The prediction equation is the equation (4):

$$
p\left(x_{k} \mid z_{1: k-1}\right)=\int p\left(x_{k} \mid x_{k-1}\right) p\left(x_{k-1} \mid z_{1: k-1}\right) d x_{k-1}
$$

The posterior probability derived by priori probability, like the equation (5), (6):

$$
\begin{aligned}
& p\left(x_{k} \mid z_{1: k}\right)=\frac{p\left(z_{k} \mid x_{k}\right) p\left(x_{k} \mid z_{1: k-1}\right)}{p\left(z_{k} \mid z_{1: k-1}\right)} \\
& p\left(z_{k} \mid z_{1: k-1}\right)=\int p\left(z_{k} \mid x_{k}\right) p\left(x_{k} \mid z_{1: k-1}\right) d x_{k}
\end{aligned}
$$

The posterior probability is difficult to achieve, so a large number of sample points in the state space is used to estimate the distribution. The integration problem is converted into a summation problem using these samples, like the equation (7):

$$
p\left(x_{k} \mid z_{k}\right) \approx \sum_{i=1}^{N} \omega_{k}^{i} \delta\left(x_{k}-x_{k}^{i}\right)
$$

$\omega_{k}^{i}$ is the weight of the sample state, and the update equation is like (8):

$\omega_{k}^{i}=\omega_{k-1}^{i} \frac{p\left(z_{k} \mid x_{k}^{i}\right) p\left(x_{k}^{i} \mid x_{k-1}^{i}\right)}{q\left(x_{k}^{i} \mid x_{0: k-1}^{i}, z_{1: k}\right)}$

$q\left(x_{k}^{i} \mid x_{0: k-1}^{i}, z_{1: k}\right)$ is the importance density function.

\section{Indoor Geographic Information Structure}

To find the common topological model of the indoor structure is the key to effectively and quickly obtain GIS data in indoor map matching. The objects expressed by indoor map are as follows: corridors, rooms, bathrooms, entrances, escalators, elevators, stairs, etc. These objects are vector graphics described by points, lines, and polygons. These common elements will be used to build the abstract objects so as to provide the map data inputments for map matching. In indoor map, rooms, corridors and other objects are polygons, each polygon edge is considered to be a wall, and according to a door is on the edge of a polygon, all the polygons are divided into two categories, reachable area and unreachable area. The door is the only way to arrive the reachable area. The two abstract objects will be integrated into the properties of the particles, the properties of abstract objects is like Fig.1.

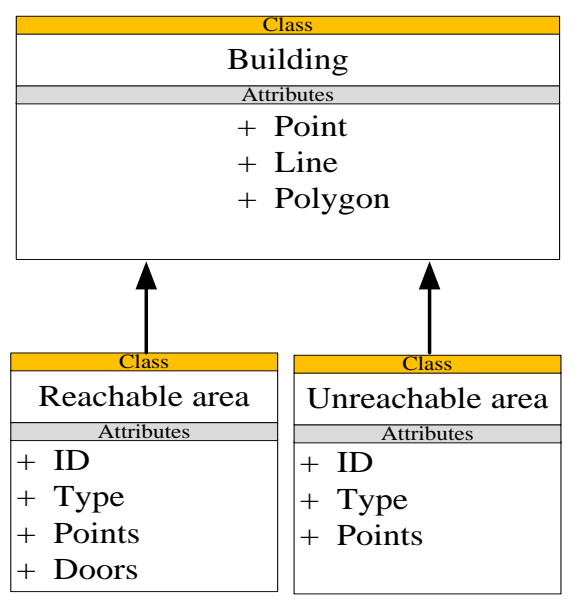

Fig. 1 Two or more references 


\section{The new map matching based on the particle filter in indoor positioning}

The state transfer equation and observation equation. The step frequency and step length have been relatively mature in PDR (Pedestrian Dead Reckoning) technology, but the pedestrian movement direction is hard to detect. The direction detection algorithm based on terminal stance was put forward in the arcticle [4]. This algorithm accurately measure the pedestrian direction under the case of pedestrian unfixed posture. This article will use this approach to be as the state transfer equation of the particle filter, like (9).

$$
X_{\mathrm{k}}=\left[\begin{array}{l}
E_{k} \\
N_{k} \\
\phi_{k}
\end{array}\right]=\left[\begin{array}{l}
E_{k-1}+L_{k} \cdot \sin \left(\phi_{\mathrm{k}}\right) \\
N_{k-1}+L_{k} \cdot \cos \left(\phi_{\mathrm{k}}\right) \\
\phi_{\mathrm{k}-1}+\Delta \phi_{\mathrm{k}}
\end{array}\right]
$$

The observation equation was given by indoor wireless positioning results, PDR results and error range, like (10).

$$
X_{\mathrm{k}}=\left[\begin{array}{l}
E_{k} \\
N_{k} \\
L_{\mathrm{k}} \\
\phi_{k}
\end{array}\right]+\left[\begin{array}{l}
\delta_{e} \\
\delta_{n} \\
\delta_{l} \\
\delta_{\phi}
\end{array}\right]
$$

$E_{k}$ is the k-th east coordinate, and $N_{k}$ is the k-th north coordinate. $L_{\mathrm{k}}$ is the k-th step length, and $\phi_{k}$ is k-th pedestrian direction.

The weights of particles fused the indoor geographic information. Indoor wireless positioning system can be viewed as a discrete system. Each predicted particle will be get by the state transfer equation, besides, the more close to the real state of the particles, the observations are more likely to get. Therefore, each particle needs to be given a right weight value. The weights sum of all particles can be expressed positioning state estimation. The particle weight calculation this paper proposed is as follows:

1 The weight of particle in reachable and unreachable area, like (11).

$$
\mathrm{w}_{r}=\left\{\begin{array}{l}
0, \text { Ineffective Particle } \\
W_{\text {reach }}, \text { Particle in reachable area }
\end{array}\right.
$$

The particle in unreachable area is considered as ineffective particle, and its weight is set to 0 . The weight of particle in reachable area is set to $W_{\text {reach }}$.

2 The weight of particle crossing the barrier or not, like (12).

$$
\mathrm{w}_{\mathrm{c}}=\left\{\begin{array}{l}
0, \text { Crossed the barrier } \\
W_{\text {normal }}, \text { Through the door }
\end{array}\right.
$$

If the particles cross the barrier by the door, it is normal, and $W_{\text {normal }}$ is the weight. When the particles cross the barrier which no doors on it are considered as unnormal, and the weight is set 0 . 3 The distance weight of particles at neighboring time, like (13).

$$
\mathrm{w}_{d}=\left\{\begin{array}{l}
0, \text { dis }>S \\
S-\text { dis }, 0 \leq \text { dis } \leq S
\end{array}\right.
$$

dis is the distance between the two particles at neighboring time, $S$ is the step length observation at this time.

4 The direction weight of particles at neighboring time, like (14).

$$
\omega_{\theta}=\left\{\begin{array}{l}
0, \theta>\xi \\
\cos \theta, \theta \leq \xi
\end{array}\right.
$$


$\theta$ is the difference between the direction of the particle at current time and the line combined with two particles at the neighboring time. $\xi$ is the angle difference threshold.

The sum of this four weight values will used to estimate the best result, like (15).

$$
\mathrm{w}_{k}^{i}=\mathrm{w}_{\mathrm{r}}^{i}+w_{c}^{i}+w_{d}^{i}+w_{\theta}^{i}, i=1, . ., N
$$

Resampling stage. To prevent particles from degradation, resampling is needed based on the importance sampling, copying the large weight particles, and eliminating the small weight particles. First of all, the normalization of weights is like (16).

$$
\mathrm{w}_{k}^{i}=\frac{w_{k}^{i}}{\sum_{i=1}^{N} w_{k}^{i}}
$$

After the normalization, the true state estimation can be obtained, like (17).

$$
\hat{P}=\sum_{i}^{N} p_{i} \cdot w_{i}
$$

Experimental Validation and Analysis. Experiments was based on the android system terminal, the original indoor positioning results were obtained by Wi-Fi positioning technology, and the sensors used the electronic compass and accelerometer, and the electronic map used the high-precision indoor relative coordinate system.

In Fig. 2, Particles distribution before updating is displayed. The blue and light blue points represent the particles distribution at the k-1 time. The black and navy blue points represent the particles distribution at the $\mathrm{k}$ time. The blue and purple points in the gas room are viewed as ineffective particles, because the gas room with no doors on its edge is unreachable area.

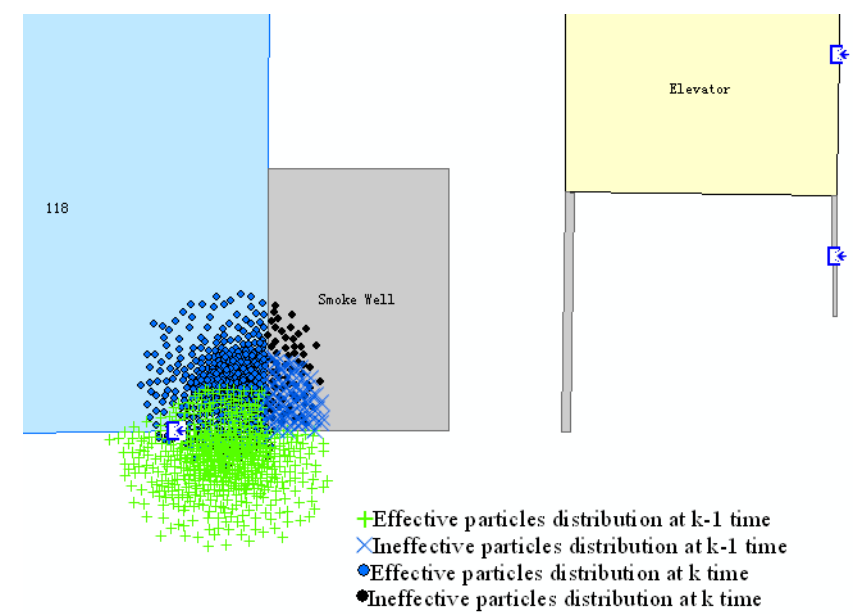

Fig. 2 Particles distribution before the particle updating

After the particle updating based on each parcticle weight value, ineffective particles will be removed, in Fig. 3. 


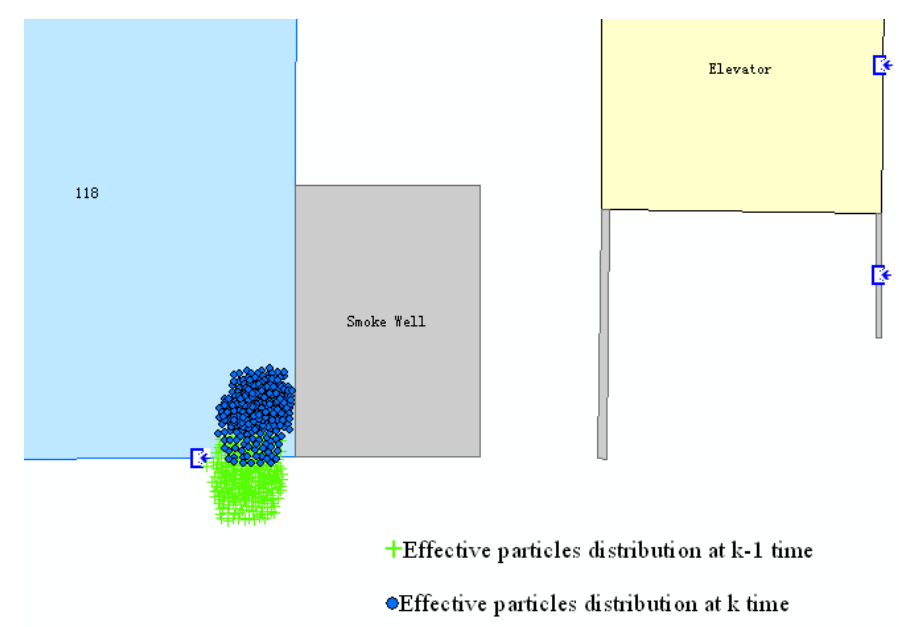

Fig. 3 Particles distribution after the particle updating

After a period of positioning, as shown in Fig. 4, the positioning track of pedestrians is displayed. In the figure, the blue points are the original wireless location, and the green points are the particle filter results with only sensor information. The red points are the particle filter results fused with map and sensor information. As can be seen from the figure, the original positioning results displayed on the map more chaos, causing poor user experience, and only fusing sensor information, the phenomena that crossing the wall often occur because there is no map of constraint. However, by adding the map information after the particle filter, the positioning results are further corrected, and the phenomena that crossing the wall or falling into the wrong room does not occur. A large number of indoor walking experiments show that this map-matching algorithm can make the error of indoor positioning less than $2.2 \mathrm{~m}$ at the $93 \%$ of the time.

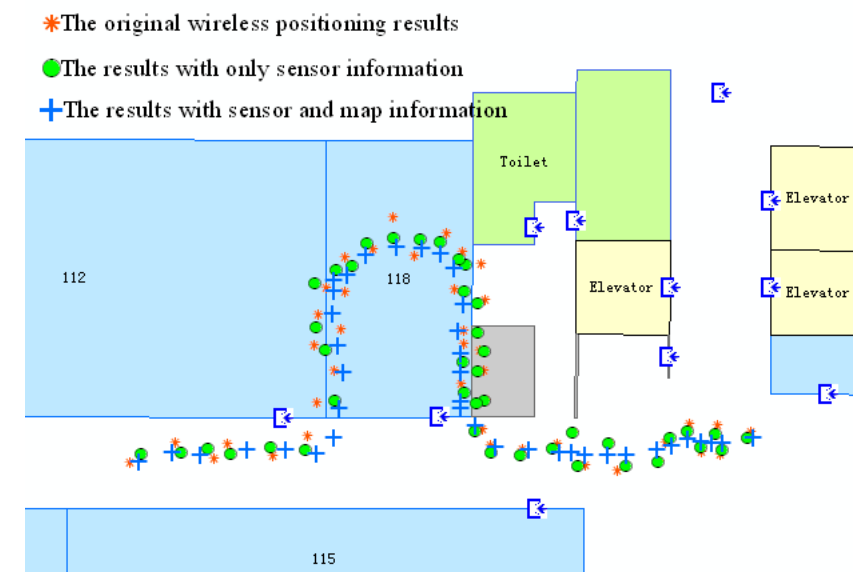

Fig. 4 The positioning track of pedestrian with different situations

\section{Summary}

In the indoor positioning, wireless positioning accuracy is not guaranteed positioning results falling into the correct area, and this can cause poor user experience. Based on the particle filter, the indoor map-matching algorithm is proposed fused with the map and sensor information, to improve indoor positioning accuracy and user experience degree.

\section{Acknowledgements}

This work is supported by the National Nature Science Foundation of China (No.61372110) and the National High Technology Research and Development Program of China (863 Program)(No. 20 12AA120801). The funders had no role in study design, data collection and analysis, decision to pu blish, or preparation of the manuscript. 


\section{References}

[1] Deng Zhonglinag, Yu Yanpei, Yuan Xie. Situation and Development Tendency of Indoor Positioning [J]. China Communications, 2013, 3: 42-55.

[2] Davidson P, Collin J, Takala J. Application of particle filters for indoor positioning using floor plans[C]. Ubiquitous Positioning Indoor Navigation and Location Based Service (UPINLBS), 2010. IEEE, 2010: 1-4.

[3] Arulampalam M S, Maskell S, Gordon N, et al. A tutorial on particle filters for online nonlinear. non-Gaussian Bayesian tracking[J]. Signal Processing, IEEE Transactions on, 2002, 50(2): 174-188.

[4] Keji Wang, Zhongliang Deng, et al.Pedestrian dead reckoning in handheld terminal with inertial measurement unit[C]. China Satellite Navigation Conference (CSNC) 2014 Proceedings: Volume III Lecture Notes in Electrical Engineering Volume 305, 2014, pp 681-690. 